\title{
THE OPTICAL PROPERTIES OF CARBON AND GRAPHITE DEPEND ON CRYSTAL STRUCTURE AND TEMPERATURE.
}

\author{
C. KOIKE(1), H. SHIBAI(2), T. TANABE(3), H. SUTO(4) // AND \\ H.SOGAWA(5), C. KAITO(6),Y. ANDO(7), M. FUKADA(1) \\ (1)Kyoto Pharmaceutical Univ.,Kyoto 607, (2)Nagoya Univ., \\ Nagoya 464-01, (3)Inst.of Astronomy, the Univ.of Tokyo, Tokyo \\ 181, (4)National Astronomical Observ., Tokyo 181, (5)Kyoto \\ Univ.,Kyoto 606, (6)Ritumeikan Univ.,Shiga 525, (7)Meijyou \\ Univ.,Nagoya 464
}

We measured the optical properties of various carbon and graphite in wide wavelength from UV to FIR. The correlation between the optical properties at UV and MIR and the degree of crystallization has not been so clear. The correlation between the spectral index at FIR and the degree of crystallization has been clear.The spectral index increases from 0.6 to 1.3 for carbon samples, and from 1.8 to 2.9 for graphite samples. The spectral index of carbon samples increases as the size of microcrystallites increase and crystal structure changes to onion-like. The spectral index of graphite samples increases as the structures of graphite crystal become perfect. These measurements were made at room temperature. Neverthless, the carbonaceous dust grains might be affected by surrounding temperature. So, we measured the spectra at low temperature (He liquid temperature). All samples of amorphous carbon at liquid He temperature show larger spectral index than that at room temperature. The sample of graphite T10, the best crystal structure among samples, shows the same spectral index as at liquid $\mathrm{He}$ and room temperature. Another samples of graphite at liquid He show nearly same spectral index as at room temperature. The spectral index in the FIR strongly depends on the degree of crystallization of carbon and graphite samples, and surrounding temperature. We can estimate the degree of crystallization from the spectral index of the absorption of carbon or graphite samples, and furthermore, from the observed index of circumstellar dust around carbon stars. This fact might give the clue to know the thermal history of carbonaceous dusts in the solar system and circumstellar around primitive stars. 